\title{
Mediastinal Rhabdomyosarcoma
}

National Cancer Institute

\section{Source}

National Cancer Institute. Mediastinal Rhabdomyosarcoma. NCI Thesaurus. Code C6617.

A malignant mesenchymal tumor with skeletal muscle differentiation affecting the mediastinum. 